is Research Square

\title{
Nurses' Awareness and Stress on Perinatal Bereavement Care
}

\section{Eunhui Kim}

Seoul National University Bundang Hospital

Hae Won Kim ( $\square$ haewon@snu.ac.kr)

seoul national university https://orcid.org/0000-0002-6730-1575

\section{Research article}

Keywords: Perinatal death, bereavement, attitude

Posted Date: September 15th, 2020

DOI: https://doi.org/10.21203/rs.3.rs-60994/v1

License: (c) This work is licensed under a Creative Commons Attribution 4.0 International License. Read Full License 


\section{Abstract}

Background: A descriptive study was conducted to examine nurses' awareness and stress related to perinatal bereavement care (PBC), improving nurses' capabilities related to PBC.

Methods: 136 nurses who had experienced perinatal death at least once participated. Data were analyzed using descriptive statistics in SPSS version 25.0 for Windows. Nurses' awareness was assessed in terms of three subdomains (attitudes, importance of policies related to PBC, and importance of training related to $\mathrm{PBC}$ ).

Results: The participants high scores for the attitude-related items of "giving sufficient time to bereaved parents to mourn for their dead baby" (4.54 points) and "nurses should treat bereaved parents with respect and dignity" (4.51 points), and they perceived a high level of importance for the policy-related items of "every staff member in the hospital should understand the policies relevant for PBC" and "when nurses feel emotional exhaustion, they should seek support" (4.58 points). Nurses' stress was high when performing PBC in combination with a heavy workload (4.25 points) and when informing parents of a poor prognosis (4.25 points). Nurses' attitude toward PBC was associated with the perceived importance of policies $(r=0.40, p<.001)$, the perceived importance of PBC-related training $(r=0.61, p<.001)$, and stress related to PBC $(r=0.29, p<.001)$.

Conclusions: Establishing hospital policies related to PBC and providing PBC training for nurses could positively affect nurses' attitudes toward PBC. A stress management program for nurses could reduce the stress caused by PBC.

\section{Background}

The perinatal mortality rate, which is an index that reflects the public health and health status of mothers and newborns [1], was 3.5 per 1,000 total births in 2009 (total number, 1,546) and 2.8 per 1,000 total births in 2018 in South Korea (total number, 904), but the rate increases as mothers become older [2]. Although several definitions are used for perinatal death based on the time of fetal death and postnatal death, the most common definition includes deaths at more than 20 weeks of gestation and death within 28 days of birth [3].

Nurses are the closest caregivers to parents who experience perinatal death and are perceived as the most helpful medical staff [4]. Nurses' perceptions of caring activities related to perinatal death are related to their attitudes and education, as well as institutional policies [5]. That is, if a nurse has negative attitudes (e.g., fear and frustration) when treating a family who has suffered the death of a baby, it can result in a negative attitude toward the performance of care [6]. In contrast, knowledge and expertise with interventions related to parental mourning counseling can improve empathy and comprehension, helping to foster a positive nursing attitude that is helpful when performing actual nursing activities [5]. Nurses are also influenced by the existence of policies and protocols related to death and nursing in the workplace [7]. Maintaining high-quality policies could help to build a mourning culture to support parents' and family members' experiences of bereavement [8]. In addition, when a baby dies in the hospital, nurses experience extreme stress when providing support for the parents or family [9-11]. Nurses feel guilty under these circumstances [12], and persistent stress in nurses associated with perinatal death could negatively affect their perceptions of caring for patients affected perinatal death [13].

Several studies on nurses' attitudes, perceptions, and stress related to perinatal bereavement care (PBC) have been conducted [10, 14-16], but no studies have investigated this topic in Korea. Therefore, this study examined nurses' awareness and stress related to PBC. These parameters were hypothesized to be correlated. This study therefore aimed to provide basic data for improving nurses' capabilities related to PBC.

\section{Definition of terminology}

1) Perinatal death has been defined as an involuntary loss of pregnancy due to stillbirth (more than 20 weeks), or the death of a newborn within 28 days after delivery $[17,18]$.

\section{Methods}

\section{Research Design}

This descriptive correlational research was conducted to identify nurses' awareness and stress related to PBC and to examine their relationships.

\section{Research Participants}


The participants of this study were nurses working at general hospitals in departments that deal with perinatal death. Participants were recruited through convenience sampling. The inclusion criteria were as follows: nurses working in the obstetrics and pediatric nursing units who had experienced at least one case of perinatal death. Nurses with less than 1 year of experience were excluded because perinatal bereavement was expected to be an infrequent experience. The sample size was calculated using G*Power version 3.10 , with a significance level of 0.05 , power of 0.90 , and a moderate effect size of 0.3 . The required number of samples was determined to be 109 . The questionnaire was distributed to a total of 150 nurses, considering a possible dropout rate of $20 \%$, and 148 participants participated in the study. Twelve responses were incomplete, so 136 questionnaires were ultimately analyzed.

\section{Research Tools}

\section{Nurses' Awareness of PBC}

After translation, the tool developed by Chan et al. [5] (Nurses' Attitudes towards Perinatal Bereavement Support; NAPBS) was used in this study (supplementary file 1). This self-reporting tool contains a total of 25 questions that respondents answer on a 5-point Likert score, ranging from "I do not agree at all" to "I strongly agree." This tool consists of three subdomains: attitude (13 items), importance of policies related to PBC (4 items), and importance of training related to PBC (8 items). Higher scores correspond to more positive attitudes toward PBC or a greater recognition of the importance of policies or training related to PBC. Chan et al. [5] reported that Cronbach's a was .92 for the total items, and $.86, .83, .90$. for the three subdomains of attitude, importance of policies, and importance of training, respectively. In the current study, Cronbach's a was .87 for the total items, and $.73, .67$, and .90 . for the three subdomains, respectively.

\section{Nurses' Stress Related to PBC}

The tool developed by Jang was used in this study after revision (supplementary file 2)[19]. This tool is composed of 29 questions in four domains, consisting of 6 items on difficulties in providing care for patients affected by perinatal death, 5 items on lack of knowledge, 10 items on inadequacies of the environment and systems for handling perinatal death, and 8 items on psychological difficulties. The items in this tool are answered using a 5-point Likert score from "I do not agree at all (1 point)" to "I strongly agree (5 points)," with higher scores indicating high levels of stress. For all items, Jang [19] reported that Cronbach's a was .87, while in the current study, Cronbach's a was .89.

\section{General Characteristics}

The questionnaires were developed in this study (supplementary file 3). Information was gathered on participants' demographic characteristics, including their level of education, whether they were religious, and their marital status. Additionally, information was collected on their personal experiences, such as their workplace, total career experience and experience at their current workplace, personal bereavement experience, and experiences of PBC. They were also asked about whether they had received training on PBC. These factors were hypothesized to be associated with nurses' perceptions and stress related to PBC.

\section{Research Procedures}

Author's Approval for Use of the Research Tool

The developer of the NAPBS tool, Moon-Fai Chan, approved its use in this study.

\section{Forward Translation}

The original tool was first translated into Korean by a translation expert at the Language Education Center of OO University. The translation was then reviewed by three experts (one nurse who is a native speaker of Korean and fluent in both Korean and English, as well as two maternity nursing professors). They determined whether there was any need for corrections due to the accuracy of the initial translation and cultural differences. The translation did not focus on translating individual words and their meanings into Korean; instead, the core concepts were emphasized [20], with the goal that the end product would not feel like a translated tool. As such, it was adjusted to fit the socio-cultural situation and medical institutions in Korea

\section{Reverse Translation}


A written translation was again conducted (reverse translation) into English by the nurse. The reverse translator is fluent in both Korean and English, and is a nurse currently working in the United States. The previous three experts who participated in the first translation reviewed the equivalence between the reverse translation and the original version to finalize the translation.

\section{Review of Expert Committee Members (content validity)}

The content validity of the translated tool was examined by 10 nursing experts. The survey was conducted once from July 1 to July 15 . 2019 . The experts included one nursing professor, four head nurses with 10 years or more of experience in the delivery room or neonatal intensive care unit, and five staff nurses with 7 years or more in the relevant departments mentioned above.

They were asked to assess the validity of each item as 1 point for "never valid," 2 points for "not valid," 3 points for "valid," and 4 points for "very valid." All 25 items had a content validity index of 0.8 or higher, and were selected for inclusion in the final version.

\section{Preliminary Study}

Prior to study, the translated Korean version of the NAPBS was preliminarily tested among 10 nurses working at hospitals located in Seoul and Gyeonggi Province. Thereafter, the Korean version of the tool was finalized.

\section{Data Collection}

After receiving IRB approval from S University Hospital (IRB No. B-1908/561-305), data were collected from September 1 to September 31, 2019. After obtaining permission from officials at seven general hospitals in Seoul and Gyeonggi-do, where the data were collected, potential participants received an explanation about the study purpose and procedures with a written protocol, and a questionnaire was distributed to nurses who voluntarily chose to participate. The participants provided written informed consent, and the collected data were encrypted and processed in a way that respected the confidentiality of the participants.

\section{Data analysis}

Data were analyzed using SPSS for Windows version 25.0 (IBM Corp., Armonk, NY, USA). The specific analytical methods were as follows.

1. General characteristics and the main variables of the study were analyzed using descriptive statistics (mean, standard deviation, frequency, and percentage).

2. Nurses' awareness (attitude, importance of policies, and importance of training related to PBC) and PBC-related stress according to their general characteristics were analyzed using the independent t-test and one-way analysis of variance. If necessary, the Scheffé test was conducted as a post hoc test.

3. The relationships among the three subdomains of awareness and stress were analyzed by Pearson correlation coefficients. All tests used a significance level of 0.05 .

\section{Results}

\section{General Characteristics of the Participants}

The average age of the nurses was 31.33 ( \pm 6.76 ) years old, with the age group of $25-29$ years old comprising $44.9 \%$ of all participants. All of the participants were women, $35.3 \%$ were married, $19.9 \%$ responded that they had children, and $58.1 \%$ were not religious. The majority ( $77.2 \%$ ) had a bachelor's degree, and $14.0 \%$ stated that they had a master's degree or higher.

Recent experiences (within the past year) of bereavement of a close friend or family member was reported by $16.2 \%$ of participants. $7.4 \%$ had previously received education on bereavement care, and $50.7 \%$ had no policy related to bereavement care in their current workplace.

Overall, $82.4 \%$ of participants were staff nurses, $47.1 \%$ worked in the delivery room, and $44.1 \%$ worked in the neonatal intensive care unit. On average, nurses' career duration was 100.17 ( \pm 81.93) months (range, 12 months to 396 months) and $50.0 \%$ had at least 73 months of career experience. The reported frequency of caring of parents with bereavement was once every 3 months for $33.8 \%$ of respondents, once a year for $26.5 \%$, and once a month for $21.3 \%$. In terms of the total number of instances of PBC experienced by participants, $33.1 \%$ reported having

Page $4 / 16$ 
experienced fewer than 5 instances, $31.6 \%$ reported having experienced 15 or more instances, and $20.6 \%$ of participants reported having experienced PBC 5-9 times (Table 1). 


\begin{tabular}{|c|c|c|c|}
\hline Characteristics & Categories & $N(\%)$ & $\mathrm{M} \pm \mathrm{SD}$ \\
\hline \multirow[t]{5}{*}{ Age (years) } & Range: $23-55$ & & $31.33 \pm 6.73$ \\
\hline & $20-24$ & $11(8.1)$ & \\
\hline & $25-29$ & $61(44.9)$ & \\
\hline & $30-34$ & $29(21.3)$ & \\
\hline & $\geq 35$ & $35(25.7)$ & \\
\hline Gender & Female & 136(100.0) & \\
\hline \multirow[t]{4}{*}{ Religion } & Protestantism & $29(21.3)$ & \\
\hline & Catholicism & 16(11.8) & \\
\hline & Buddhism & $12(8.8)$ & \\
\hline & None & $79(58.1)$ & \\
\hline \multirow[t]{2}{*}{ Marital status } & Unmarried & $88(64.7)$ & \\
\hline & Married & $48(35.3)$ & \\
\hline \multirow[t]{2}{*}{ Having children } & Yes & $27(19.9)$ & \\
\hline & No & $7(5.1)$ & \\
\hline \multirow[t]{3}{*}{ Education } & Associate's & $12(8.8)$ & \\
\hline & Bachelor's & 105(77.2) & \\
\hline & Master's & $19(14.0)$ & \\
\hline \multirow[t]{2}{*}{ Bereavement experience in the past year } & Yes & $22(16.2)$ & \\
\hline & No & $113(83.1)$ & \\
\hline \multirow[t]{3}{*}{ Received education about perinatal bereavement care } & Yes & $10(7.4)$ & \\
\hline & No & 124(91.2) & \\
\hline & No response & $2(1.5)$ & \\
\hline \multirow[t]{3}{*}{ Had a clear policy for the management of bereavement in the workplace } & Yes & $61(44.9)$ & \\
\hline & No & $69(50.7)$ & \\
\hline & No response & $6(4.4)$ & \\
\hline \multirow[t]{3}{*}{ Position in the clinical field } & Staff Nurse & 112(82.4) & \\
\hline & APN & $4(2.9)$ & \\
\hline & Charge nurse & $20(14.7)$ & \\
\hline \multirow[t]{4}{*}{ Current working unit } & Delivery room & $64(47.1)$ & \\
\hline & $\mathrm{NICU}$ & $60(44.1)$ & \\
\hline & Nursery & $4(2.9)$ & \\
\hline & Others & $8(5.9)$ & \\
\hline \multirow[t]{4}{*}{ Clinical career (months) } & Range: $12-396$ & & $100.16 \pm 81.93$ \\
\hline & $\leq 24$ & 19(14.0) & \\
\hline & $25-48$ & $20(14.7)$ & \\
\hline & $49-72$ & $29(21.3)$ & \\
\hline
\end{tabular}

$M$ mean, NICU Neonatal intensive care unit, SD Standard deviation, APN advanced practice nurse 


\begin{tabular}{|c|c|c|c|}
\hline Characteristics & Categories & $N(\%)$ & $\mathrm{M} \pm \mathrm{SD}$ \\
\hline & $\geq 73$ & $68(50.0)$ & \\
\hline \multirow[t]{5}{*}{ Clinical career in the obstetrics or pediatrics unit (months) } & Range: 5-319 & & $73.44 \pm 58.39$ \\
\hline & $\leq 24$ & 27(19.9) & \\
\hline & $25-48$ & $28(20.6)$ & \\
\hline & $49-72$ & $28(20.6)$ & \\
\hline & $\geq 73$ & $52(39.0)$ & \\
\hline \multirow{6}{*}{ Frequency of facing circumstances involving bereaved parents (times) } & Less than once a year & $19(14.0)$ & \\
\hline & Once a year & $36(26.5)$ & \\
\hline & Once a every 3 months & $46(33.8)$ & \\
\hline & Once a month & $29(21.3)$ & \\
\hline & Once a week & $3(2.2)$ & \\
\hline & Irregular & $3(2.2)$ & \\
\hline \multirow[t]{4}{*}{ Total number of experiences of perinatal death (times) } & $<5$ & $45(33.1)$ & \\
\hline & $5-9$ & $28(20.6)$ & \\
\hline & $10-14$ & $20(14.7)$ & \\
\hline & $\geq 15$ & 43(31.6) & \\
\hline
\end{tabular}

\section{Nurses' Awareness and Stress Related to PBC}

The average scores for the three subdomains of nurses' awareness were $52.59( \pm 4.94)$ points for attitude toward PBC, $15.86( \pm 2.03)$ points for the importance of policies related to PBC, and $34.72( \pm 3.70)$ points for the importance of training on PBC. Specifically, the highest scores were reported for "It is important to find support when feeling emotional exhaustion" (4.58 points), "I think we need enough time for bereaved parents to mourn" (4.54 points), and "I will treat bereaved parents with respect and dignity" (4.51 points) (Table 2). 
I believe that a grief counseling program can provide psychological support to the bereaved couple.

I agree that the Good-bye Baby parent support group provides support to parents with similar experience.

All those who care for and support bereaved parents should have access to support for themselves.

Nurses should feel assured that they are working within an operational policy which is adequate and appropriate.

The unit should have a clear policy for the management of bereavement.

The policy should be clearly informed to all staff involved.

The policy should be understood by all staff involved.

Nurses involved in the care and support of bereaved parents need to be equipped with relevant knowledge, skills, and understanding.

Nurses need to feel confident that they are providing adequate and appropriate care.

Nurses need to know that they have limitation when provided perinatal bereavement care.

$M$ mean, SD Standard deviation

Regarding the nurses' stress, the overall average score was 112.16 ( \pm 13.46$)$ points. The items with the highest scores were "caring for a dying newborn along with a heavy workload" (4.25 points) and "telling parents that their neonate has a poor prognosis" (4.25 points) (Table 3 ). 
Table 3

Descriptive Analysis of Nurses' Stress in Caring for Patients Affected by Perinatal Death ( $N=136)$

\begin{tabular}{|c|c|}
\hline Contents & $\mathrm{M} \pm \mathrm{SD}$ \\
\hline Lack of knowledge & $19.80 \pm 2.87$ \\
\hline When you cannot give emotional support to bereaved parents due to a lack of communication skills & $4.14 \pm 0.72$ \\
\hline When an emergency situation cannot be handled quickly & $4.08 \pm 0.96$ \\
\hline When it is difficult to give systematic care for a dying baby & $3.93 \pm 0.80$ \\
\hline When knowledge on terminal care is not sufficient & $3.88 \pm 0.80$ \\
\hline When the treatment and nursing care of a dying baby is not timely & $3.77 \pm 0.91$ \\
\hline Lack of an appropriate physical and structural environment & $39.25 \pm 5.73$ \\
\hline When talking to bereaved parents about the poor prognosis of a baby & $4.25 \pm 0.71$ \\
\hline When administrative work is delayed after a death of baby & $4.03 \pm 0.90$ \\
\hline When turning away from the sadness of bereaved parents & $4.01 \pm 0.83$ \\
\hline When there is no guideline or policy for bereavement care & $3.98 \pm 0.84$ \\
\hline When bereaved parents do not have enough time spent with their dying baby & $3.97 \pm 0.80$ \\
\hline When it is difficult to give a private room to bereaved parents & $3.95 \pm 0.83$ \\
\hline When having to explain the administrative work of funeral procedures after death & $3.82 \pm 1.07$ \\
\hline When parents want ongoing life-sustaining treatment, even if the baby's condition is hopeless & $3.80 \pm 1.02$ \\
\hline When bereaved parents do not accept their baby's death after a doctor declares it & $3.76 \pm 1.07$ \\
\hline When parents do not make any decision about a dying baby's care plan & $3.68 \pm 0.92$ \\
\hline Difficulties related to end-of life care practice & $23.46 \pm 3.72$ \\
\hline When you have to care for a dying baby with a heavy workload & $4.25 \pm 0.83$ \\
\hline When carrying out post-mortem treatment directly & $4.07 \pm 1.01$ \\
\hline When caring for dying babies frequently & $4.04 \pm 0.85$ \\
\hline When a terminal baby's care brings a work overload & $3.90 \pm 1.06$ \\
\hline When nursing a dying baby is physically exhausting due to excessive physical labor & $3.79 \pm 1.04$ \\
\hline When bereaved parents have a lot of requirements when a baby is about to die & $3.41 \pm 1.16$ \\
\hline Emotional stress & $39.25 \pm 5.73$ \\
\hline When a long-term nursing baby dies & $4.06 \pm 0.94$ \\
\hline When you think you would experience a dying baby again & $3.97 \pm 1.05$ \\
\hline When caring for another baby before grieving for a baby & $3.82 \pm 0.99$ \\
\hline When your feelings become dull as you experience repeated death & $3.80 \pm 0.99$ \\
\hline When you need to perform temporary symptomatic nursing care and not care for a therapeutic purpose & $3.63 \pm 0.86$ \\
\hline When trying to forget a dying baby, but not finding it easy & $3.52 \pm 0.99$ \\
\hline When you cannot talk about your feelings after experiencing the death of a baby & $3.49 \pm 0.94$ \\
\hline When a baby's death is felt to result from the nurse's own failure & $3.37 \pm 1.26$ \\
\hline Total & $112.16 \pm 13.46$ \\
\hline
\end{tabular}




\section{Awareness and Stress Related to PBC by General Characteristics}

Attitude toward PBC showed significant associations with having received training on PBC $(\mathrm{t}=-3.38, p=.003)$ and length of career experience in obstetrics and pediatric units $(r=.22, p=.005)$. The importance of policies related to PBC showed significant associations with the existence of a policy in the workplace $(\mathrm{t}=-.423, p=.005)$, the number of experiences of PBC $(\mathrm{F}=4.47, p=.005)$, and length of career experience in related units $(r=.19, p=.015)$. The importance of training for PBC was associated with length of career experience in related units $(r=.16, p=.029)$, and the number of experiences of PBC $(F=4.15, p=.008)$.

However, no factor showed a significant relationship with nurses' PBC-related stress (Table 4). 
Table 4

Differences in Nurses' Awareness and Stress when Caring for Patients Affected by Perinatal Death according to General Characteristics ( $\mathrm{N}=$ 136)

\begin{tabular}{|c|c|c|c|c|c|c|c|c|c|c|c|c|c|}
\hline \multirow[t]{2}{*}{ Characteristics } & \multirow[t]{2}{*}{ Categories } & \multicolumn{3}{|c|}{ Attitude towards PBC } & \multicolumn{3}{|c|}{$\begin{array}{l}\text { Perceived importance } \\
\text { of policies on PBC }\end{array}$} & \multicolumn{3}{|c|}{$\begin{array}{l}\text { Perceived } \\
\text { importance of } \\
\text { training for } \\
\text { PBC }\end{array}$} & \multicolumn{3}{|c|}{$\begin{array}{l}\text { Nurses' stress related to } \\
\text { PBC }\end{array}$} \\
\hline & & $\begin{array}{l}M \pm \\
S D\end{array}$ & $\begin{array}{l}\text { ror } t \\
\text { or } F\end{array}$ & $p$ & $\begin{array}{l}M \pm \\
S D\end{array}$ & $\begin{array}{l}\text { ror } t \\
\text { or } F\end{array}$ & $p$ & $\begin{array}{l}M \pm \\
S D\end{array}$ & $\begin{array}{l}\text { ror } t \\
\text { or } F\end{array}$ & $p$ & $M \pm S D$ & $\begin{array}{l}\text { ror } t \\
\text { or } F\end{array}$ & $p$ \\
\hline Age (year) & & & .13 & .064 & & .02 & .403 & & -.04 & .323 & & .01 & .452 \\
\hline \multirow[t]{2}{*}{ Religion } & Yes & $\begin{array}{l}53.43 \\
\pm 5.30\end{array}$ & \multirow[t]{2}{*}{1.67} & \multirow[t]{2}{*}{.097} & $\begin{array}{l}15.75 \\
\pm 2.24\end{array}$ & \multirow[t]{2}{*}{-0.53} & \multirow[t]{2}{*}{.599} & $\begin{array}{l}35.30 \\
\pm 3.91\end{array}$ & \multirow[t]{2}{*}{1.55} & \multirow[t]{2}{*}{.124} & $\begin{array}{l}113.38 \\
\pm 14.34\end{array}$ & \multirow[t]{2}{*}{0.88} & \multirow[t]{2}{*}{.381} \\
\hline & No & $\begin{array}{l}52.00 \\
\pm 4.61\end{array}$ & & & $\begin{array}{l}15.93 \\
\pm 1.88\end{array}$ & & & $\begin{array}{l}34.31 \\
\pm 3.51\end{array}$ & & & $\begin{array}{l}111.31 \\
\pm 12.83\end{array}$ & & \\
\hline \multirow[t]{2}{*}{ Marital status } & Unmarried & $\begin{array}{l}52.07 \\
\pm 4.48\end{array}$ & \multirow[t]{2}{*}{1.67} & \multirow[t]{2}{*}{.097} & $\begin{array}{l}15.86 \\
\pm 2.11\end{array}$ & \multirow[t]{2}{*}{-0.03} & \multirow[t]{2}{*}{.979} & $\begin{array}{l}34.69 \\
\pm 3.48\end{array}$ & \multirow[t]{2}{*}{0.12} & \multirow[t]{2}{*}{.907} & $\begin{array}{l}111.65 \\
\pm 12.91\end{array}$ & \multirow[t]{2}{*}{0.60} & \multirow[t]{2}{*}{.548} \\
\hline & Married & $\begin{array}{l}53.54 \\
\pm 5.61\end{array}$ & & & $\begin{array}{l}15.85 \\
\pm 1.90\end{array}$ & & & $\begin{array}{l}34.77 \\
\pm 4.10\end{array}$ & & & $\begin{array}{l}113.10 \\
\pm 14.50\end{array}$ & & \\
\hline \multirow[t]{3}{*}{ Education } & Associate's & $\begin{array}{l}52.50 \\
\pm 5.99\end{array}$ & \multirow[t]{3}{*}{0.49} & \multirow[t]{3}{*}{.615} & $\begin{array}{l}16.08 \\
\pm 2.43\end{array}$ & \multirow[t]{3}{*}{0.09} & \multirow[t]{3}{*}{.917} & $\begin{array}{l}36.25 \\
\pm 3.57\end{array}$ & \multirow[t]{3}{*}{1.53} & \multirow[t]{3}{*}{.220} & $\begin{array}{l}117.08 \\
\pm 9.86\end{array}$ & 0.93 & .398 \\
\hline & Bachelor's & $\begin{array}{l}52.41 \\
\pm 4.86\end{array}$ & & & $\begin{array}{l}15.83 \\
\pm 2.03\end{array}$ & & & $\begin{array}{l}34.45 \\
\pm 3.71\end{array}$ & & & $\begin{array}{l}111.52 \\
\pm 13.59\end{array}$ & & \\
\hline & Master's & $\begin{array}{l}53.63 \\
\pm 4.81\end{array}$ & & & $\begin{array}{l}15.89 \\
\pm 1.88\end{array}$ & & & $\begin{array}{l}35.26 \\
\pm 3.60\end{array}$ & & & $\begin{array}{l}112.58 \\
\pm 14.61\end{array}$ & & \\
\hline $\begin{array}{l}\text { Bereavement } \\
\text { experience in }\end{array}$ & Yes & $\begin{array}{l}52.74 \\
\pm 4.43\end{array}$ & -0.16 & .875 & $\begin{array}{l}15.36 \\
\pm 2.54\end{array}$ & 1.25 & .212 & $\begin{array}{l}34.95 \\
\pm 3.42\end{array}$ & -0.32 & .747 & $\begin{array}{l}113.05 \\
\pm 13.10\end{array}$ & -0.34 & .738 \\
\hline जा & No & $\begin{array}{l}52.56 \\
\pm 5.04\end{array}$ & & & $\begin{array}{l}15.96 \\
\pm 1.92\end{array}$ & & & $\begin{array}{l}34.68 \\
\pm 3.76\end{array}$ & & & $\begin{array}{l}111.99 \\
\pm 13.59\end{array}$ & & \\
\hline $\begin{array}{l}\text { Received } \\
\text { education } \\
\text { about }\end{array}$ & Yes & $\begin{array}{l}57.40 \\
\pm 3.34\end{array}$ & -3.38 & .001 & $\begin{array}{l}16.90 \\
\pm 2.47\end{array}$ & -1.69 & .094 & $\begin{array}{l}36.70 \\
\pm 3.13\end{array}$ & -1.78 & .078 & $\begin{array}{l}109.30 \\
\pm 15.20\end{array}$ & 0.64 & .522 \\
\hline $\begin{array}{l}\text { bereavement } \\
\text { care }\end{array}$ & No & $\begin{array}{l}52.14 \\
\pm 4.82\end{array}$ & & & $\begin{array}{l}15.79 \\
\pm 1.96\end{array}$ & & & $\begin{array}{l}34.54 \\
\pm 3.72\end{array}$ & & & $\begin{array}{l}112.14 \\
\pm 13.30\end{array}$ & & \\
\hline $\begin{array}{l}\text { Had a clear } \\
\text { policy for the } \\
\text { management }\end{array}$ & Yes & $\begin{array}{l}53.14 \\
\pm 4.99\end{array}$ & -1.53 & .129 & $\begin{array}{l}16.52 \\
\pm 1.79\end{array}$ & -4.23 & $<.001$ & $\begin{array}{l}35.26 \\
\pm 3.60\end{array}$ & -1.80 & .074 & $\begin{array}{l}112.46 \\
\pm 12.56\end{array}$ & -0.54 & .587 \\
\hline $\begin{array}{l}\text { bereavement } \\
\text { in the } \\
\text { workplace }\end{array}$ & No & $\begin{array}{l}51.83 \\
\pm 4.79\end{array}$ & & & $\begin{array}{l}15.13 \\
\pm 1.95\end{array}$ & & & $\begin{array}{l}34.10 \\
\pm 3.74\end{array}$ & & & $\begin{array}{l}111.14 \\
\pm 14.35\end{array}$ & & \\
\hline $\begin{array}{l}\text { Position in the } \\
\text { clinical field }\end{array}$ & $\begin{array}{l}\text { Staff } \\
\text { Nurse }\end{array}$ & $\begin{array}{l}52.33 \\
\pm 4.64\end{array}$ & 2.07 & .130 & $\begin{array}{l}15.71 \\
\pm 2.06\end{array}$ & 1.95 & .148 & $\begin{array}{l}34.58 \\
\pm 3.53\end{array}$ & 1.26 & .288 & $\begin{array}{l}111.50 \\
\pm 12.72\end{array}$ & 1.52 & .222 \\
\hline & APN & $\begin{array}{l}57.25 \\
\pm 4.35\end{array}$ & & & $\begin{array}{l}17.25 \\
\pm 1.50\end{array}$ & & & $\begin{array}{l}37.50 \\
\pm 3.70\end{array}$ & & & $\begin{array}{l}122.75 \\
\pm 10.53\end{array}$ & & \\
\hline & $\begin{array}{l}\text { Charge } \\
\text { nurse }\end{array}$ & $\begin{array}{l}53.10 \\
\pm 6.28\end{array}$ & & & $\begin{array}{l}16.40 \\
\pm 1.82\end{array}$ & & & $\begin{array}{l}34.95 \\
\pm 4.52\end{array}$ & & & $\begin{array}{l}113.75 \\
\pm 17.23\end{array}$ & & \\
\hline $\begin{array}{l}\text { Current } \\
\text { working unit }\end{array}$ & $\begin{array}{l}\text { Delivery } \\
\text { rooma }^{a}\end{array}$ & $\begin{array}{l}51.14 \\
\pm 5.21\end{array}$ & 5.58 & .001 & $\begin{array}{l}15.70 \\
\pm 2.19\end{array}$ & 0.85 & .470 & $\begin{array}{l}34.28 \\
\pm 4.01\end{array}$ & 0.84 & .474 & $\begin{array}{l}109.98 \\
\pm 15.69\end{array}$ & 1.19 & .318 \\
\hline & $\mathrm{NICU}{ }^{\mathrm{b}}$ & $\begin{array}{l}54.14 \\
\pm 3.89\end{array}$ & & $a^{*}$ & $\begin{array}{l}15.88 \\
\pm 1.80\end{array}$ & & & $\begin{array}{l}35.15 \\
\pm 3.30\end{array}$ & & & $\begin{array}{l}113.96 \\
\pm 11.20\end{array}$ & & \\
\hline & Nursery & $\begin{array}{l}56.50 \\
\pm 5.07\end{array}$ & & & $\begin{array}{l}17.25 \\
\pm 2.75\end{array}$ & & & $\begin{array}{l}36.25 \\
\pm 5.68\end{array}$ & & & $\begin{array}{l}118.00 \\
\pm 7.75\end{array}$ & & \\
\hline & Others & $\begin{array}{l}50.62 \\
\pm 5.83\end{array}$ & & & $\begin{array}{l}16.25 \\
\pm 2.05\end{array}$ & & & $\begin{array}{l}34.25 \\
\pm 2.71\end{array}$ & & & $\begin{array}{l}113.13 \\
\pm 10.30\end{array}$ & & \\
\hline
\end{tabular}




\begin{tabular}{|c|c|c|c|c|c|c|c|c|c|c|c|c|c|}
\hline \multirow[t]{2}{*}{ Characteristics } & \multirow[t]{2}{*}{ Categories } & \multicolumn{3}{|c|}{ Attitude towards PBC } & \multicolumn{3}{|c|}{$\begin{array}{l}\text { Perceived importance } \\
\text { of policies on PBC }\end{array}$} & \multicolumn{3}{|c|}{$\begin{array}{l}\text { Perceived } \\
\text { importance of } \\
\text { training for } \\
\text { PBC }\end{array}$} & \multicolumn{3}{|c|}{$\begin{array}{l}\text { Nurses' stress related to } \\
\text { PBC }\end{array}$} \\
\hline & & $\underset{S D}{M} \pm$ & $\begin{array}{l}\text { rort } \\
\text { or } F\end{array}$ & $\mathrm{p}$ & $\underset{S D}{M} \pm$ & $\begin{array}{l}\text { rort } \\
\text { or } F\end{array}$ & $\mathrm{p}$ & $\begin{array}{l}M \pm \\
\text { SD }\end{array}$ & $\begin{array}{l}\text { rort } \\
\text { or } F\end{array}$ & $\mathbf{p}$ & $\mathrm{M} \pm \mathrm{SD}$ & $\begin{array}{l}\text { rort } \\
\text { or } F\end{array}$ & p \\
\hline $\begin{array}{l}\text { Clinical career } \\
\text { (months) }\end{array}$ & & & .12 & .089 & & .04 & .314 & & -.03 & .345 & & -.03 & .356 \\
\hline $\begin{array}{l}\text { Clinical career } \\
\text { in obstetrics } \\
\text { and pediatrics } \\
\text { units (months) }\end{array}$ & & & .22 & .005 & & .19 & .015 & & .16 & .029 & & .01 & .128 \\
\hline \multirow{6}{*}{$\begin{array}{l}\text { Frequency of } \\
\text { caring for } \\
\text { grieving } \\
\text { parents }\end{array}$} & $\begin{array}{l}\text { Less than } \\
\text { once a } \\
\text { year }\end{array}$ & $\begin{array}{l}52.91 \\
\pm 4.02\end{array}$ & 1.52 & .189 & $\begin{array}{l}15.84 \\
\pm 2.46\end{array}$ & 0.41 & .843 & $\begin{array}{l}34.47 \\
\pm 3.01\end{array}$ & 0.41 & .839 & $\begin{array}{l}114.67 \\
\pm 2.61\end{array}$ & 1.21 & .307 \\
\hline & $\begin{array}{l}\text { Once a } \\
\text { year }\end{array}$ & $\begin{array}{l}54.21 \\
\pm 4.52\end{array}$ & & & $\begin{array}{l}15.79 \\
\pm 1.91\end{array}$ & & & $\begin{array}{l}35.27 \\
\pm 3.54\end{array}$ & & & $\begin{array}{l}112.27 \\
\pm 11.97\end{array}$ & & \\
\hline & $\begin{array}{l}\text { Once every } \\
3 \text { months }\end{array}$ & $\begin{array}{l}52.61 \\
\pm 5.76\end{array}$ & & & $\begin{array}{l}15.90 \\
\pm 1.70\end{array}$ & & & $\begin{array}{l}34.73 \\
\pm 4.00\end{array}$ & & & $\begin{array}{l}111.37 \\
\pm 12.64\end{array}$ & & \\
\hline & $\begin{array}{l}\text { Once a } \\
\text { month }\end{array}$ & $\begin{array}{l}50.90 \\
\pm 4.73\end{array}$ & & & $\begin{array}{l}15.57 \\
\pm 2.38\end{array}$ & & & $\begin{array}{l}34.50 \\
\pm 3.26\end{array}$ & & & $\begin{array}{l}112.75 \\
\pm 14.85\end{array}$ & & \\
\hline & $\begin{array}{l}\text { Once a } \\
\text { week }\end{array}$ & $\begin{array}{l}51.00 \\
\pm 2.83\end{array}$ & & & $\begin{array}{l}16.00 \\
\pm 1.41\end{array}$ & & & $\begin{array}{l}36.50 \\
\pm 2.12\end{array}$ & & & $\begin{array}{l}129.00 \\
\pm 4.24\end{array}$ & & \\
\hline & Irregular & $\begin{array}{l}51.84 \\
\pm 4.34\end{array}$ & & & $\begin{array}{l}16.54 \\
\pm 2.11\end{array}$ & & & $\begin{array}{l}33.84 \\
\pm 5.16\end{array}$ & & & $\begin{array}{l}106.84 \\
\pm 18.47\end{array}$ & & \\
\hline \multirow{4}{*}{$\begin{array}{l}\text { Total number } \\
\text { of experiences } \\
\text { of } \\
\text { perinatal } \\
\text { death (times) }\end{array}$} & $<5^{\mathrm{a}}$ & $\begin{array}{l}53.79 \\
\pm 4.14\end{array}$ & \multirow[t]{4}{*}{1.96} & \multirow[t]{4}{*}{.122} & $\begin{array}{l}16.18 \\
\pm 2.08\end{array}$ & \multirow[t]{4}{*}{4.47} & \multirow[t]{4}{*}{.005} & $\begin{array}{l}35.53 \\
\pm 3.21\end{array}$ & \multirow[t]{4}{*}{4.15} & \multirow{4}{*}{$\begin{array}{l}.008 \\
a, d \\
>b^{*}\end{array}$} & $\begin{array}{l}113.93 \\
\pm 11.80\end{array}$ & \multirow[t]{4}{*}{1.43} & \multirow[t]{4}{*}{.236} \\
\hline & $5-9^{b}$ & $\begin{array}{l}50.96 \\
\pm 4.48\end{array}$ & & & $\begin{array}{l}15.11 \\
\pm 1.77\end{array}$ & & & $\begin{array}{l}32.75 \\
\pm 4.24\end{array}$ & & & $\begin{array}{l}107.86 \\
\pm 14.97\end{array}$ & & \\
\hline & $10-14^{c}$ & $\begin{array}{l}52.26 \\
\pm 5.55\end{array}$ & & & $\begin{array}{l}14.95 \\
\pm 2.14\end{array}$ & & & $\begin{array}{l}34.30 \\
\pm 3.29\end{array}$ & & & $\begin{array}{l}111.10 \\
\pm 12.98\end{array}$ & & \\
\hline & $\geq 15^{\mathrm{d}}$ & $\begin{array}{l}52.55 \\
\pm 5.50\end{array}$ & & & $\begin{array}{l}16.44 \\
\pm 1.86\end{array}$ & & & $\begin{array}{l}35.35 \\
\pm 3.58\end{array}$ & & & $\begin{array}{l}113.60 \\
\pm 14.07\end{array}$ & & \\
\hline
\end{tabular}

\section{Relationships between Nurses' Awareness and Stress Related to PBC}

The three subdomains of PBC awareness were all correlated with each other $(r=0.29-0.61)$.

Nurses' stress related to PBC was correlated with their attitude toward PBC $(r=0.29, p<.001)$ and their perceived importance of training on PBC $(r=0.38, p<.001)($ Table 5$)$. 
Table 5

Correlations of the Variables

\begin{tabular}{|llll|}
\hline & $\mathbf{1}$ & $\mathbf{2}$ & $\mathbf{3}$ \\
\hline & $\mathbf{r}(\mathbf{p})$ & & \\
\hline 1.Attitude towards perinatal bereavement care & 1 & & \\
\hline 2.Perceived importance of policies on perinatal bereavement care & 0.40 & 1 & \\
& $(<.001)$ & & \\
\hline 3.Perceived importance of training for perinatal bereavement care & 0.61 & 0.52 & 1 \\
\hline 4.Nurses' stress related to perinatal bereavement care & $(<.001)$ & $(<.001)$ & \\
& 0.29 & 0.06 & 0.38 \\
\hline
\end{tabular}

\section{Discussion}

Since $91.2 \%$ of nurses-the overwhelming majority of the participants in this study-had not received training on bereavement care, it is urgently necessary to address nurses' educational needs related to PBC.

Regarding nurses' awareness of PBC, each of the three subdomains can be considered separately. First, regarding attitudes toward PBC, nurses showed a positive attitude toward giving the parents sufficient time for the bereavement process, but they seemed to hesitate and worry about showing the dying baby to the parents. This is similar to the finding of a previous study [21] that nurses felt conflicted about whether to accept or reject parents' request to see their dead baby. However, in a systematic review, Kingdon et al. [22] reported that showing parents their dead baby and giving them the chance to hold the baby could help in the parents' bereavement process. No guideline or protocol currently exists regarding whether parents can view or photograph their dead baby in Korea; therefore, culturally-specific conversations among health professionals are needed to address this issue. Regarding policies, nurses showed a high level of recognition of the importance of all staff members understanding policies related to PBC, which supports the findings of Chan et al. [23]. Third, in relation to awareness of training, nurses placed the highest importance on seeking support when they were emotionally exhausted. This finding is similar to the results of a previous study [24], in which nurses working in the neonatal intensive care unit sought social support as their coping mechanism. Furthermore, this supports the result that nurses had the strongest demand regarding stress and exhaustion among the palliative nursing education needs [25].

In regard to nurses' stress related to PBC, the findings of high levels of stress when informing parents of a poor prognosis and coping with PBC in combination with a heavy workload are consistent with those of previous studies on nurses caring for dying adults [26-28], and neonates in the neonatal intensive care unit [19]. Therefore, administrative efforts are needed in the hospital setting to improve the efficiency of the distribution of nurses' workload and to provide spaces for parents to mourn when perinatal death occurs.

Regarding the relationships of general characteristics to nurses' awareness, it was found that previous training on PBC was related to a positive attitude toward PBC, consistent with the findings of Chan et al. [5], which emphasizes the importance of nurse training on PBC. Nurses were more aware of the importance of policies when their departments had a clear policy about bereavement care, which is similar to the finding of Wi and Kang [29], indicating that policies or protocols should be established to improve nurses' recognition and performance of bereavement care. In this study, there was an unclear relationship between the frequency of PBC and the perceived importance of training for PBC. A possible interpretation may be that as nurses came to have more experiences of PBC, they perceived PBC training as more important or valuable or became increasingly aware of the difficulties and their lack of confidence regarding PBC. Further research is needed to confirm the relationships of nursing confidence and perceived difficulties with the number of PBC experiences.

The three domains of nurses' awareness of PBC-attitude, importance of policies, and importance of training-were found to be closely related. Therefore, positive effects on the performance of PBC may occur as a result of supporting nurses' attitude and related policies and providing training or education on PBC. In addition, it appears that nurses performing PBC are vulnerable to high levels of psychological stress due to their busy workload, regardless of whether they have a positive attitude towards PBC. Medical institutions and hospitals should therefore establish clear policies and nurse support programs related to PBC. Since nursing care is continued after discharge for patients who experience perinatal death, further research on nurses working in the community setting is recommended.

The limitations of this study are as follows. First, participants were selected using convenience sampling, which could interfere with the generalizability of the results to all nurses, especially since variations exist in hospital policies, departmental characteristics, and patient 
severity. Moreover, the Korean version of the NAPBS used in this study was translated and used for the first time. Based on qualitative research into Korean nurses' awareness of PBC, the domains of the tool will be reexamined, and possibly reconstructed, in the future.

\section{Conclusions}

Nurses' awareness of PBC was higher if they had received training on perinatal death and if relevant policies had been clearly established at their workplace. More positive attitudes toward PBC were associated with higher stress. Therefore, clear policies for PBC should be implemented, and relevant education programs for nurses should be developed. In order to reduce nurses' stress related to perinatal death, institutional efforts are necessary to prevent nurses from becoming exhausted and to support nurses in the PBC.

\section{Abbreviations}

PBC: perinatal bereavement care; NAPBS: Nurses' attitude towards perinatal bereavement support; APB: advanced practice nurse

\section{Declarations}

\section{Ethics approval and consent to participate}

This study was approved by the Institutional Review Board(IRB) of Seoul National University Bundang Hospital (IRB No. B-1908-561-305). Written informed consent was obtained from the participants.

\section{Consent for publication}

Not applicable

\section{Availability of data and material}

The datasets used and analyzed during the current study are available from the corresponding author on reasonable request.

\section{Competing interests}

The authors declare no conflict of interest. The funders had no role in the design of the study; in the collection, analyses, or interpretation of data; in the writing of the manuscript; or in the decision to publish the results.

\section{Funding}

This study was supported by the National Research Foundation of Korea (NRF) grant funded by the Korea government (Grant No. 2018R1A2B2001231). The funders had no role in the design of the study; in the collection, analyses, or interpretation of data; in the writing of the manuscript; or in the decision to publish the results.

\section{Authors' contributions}

EK: Conceptualization, data collection, formal analysis, writing original darft / HK: Conceptualization, formal analysis, funding acquisition, review and editing. All authors have read and agreed to the published version of the manuscript.

\section{Acknowledgements}

We thank to the nurses participating in this study.

\section{References}

1. Cho JH, Choi SK, Chung SH, Choi YS, Bae CW. Changes in Neonatal and Perinatal Vital Statistics during Last 5 Decades in Republic of Korea: Compared with OECD Nations. Neonatal Med. 2013;20(4):402-12. http://dx.doi.org/10.5385/nm.2013.20.4.402. 
2. Statistics Korea. Infant, Maternal and Perinatal mortality Statistics. 2019. http://kosis.kr/upsHtml/online/downSrvcFile.do? PUBCODE=YJ\&FILE_NAME=/YJ/0801.hwp\&SEQ=23. Accessed July 16, 2020.

3. Barfield WD. Standard terminology for fetal, infant, and perinatal deaths. Pediatrics. 2011;128(1):177-81. https://doi.org/10.1542/peds.2011-1037.

4. Aiyelaagbe E, Scott RE, Holmes V, Lane E, Heazell AE. Assessing the quality of bereavement care after perinatal death: development and piloting of a questionnaire to assess parents' experiences. J Obstet Gynaecol. 2017;37(7):931-6. https://doi.org/10.1080/01443615.2017.1316710.

5. Chan MF, Chan SH, Day MC. Nurses' attitudes towards perinatal bereavement support in Hong Kong: a pilot study. J Clin Nurs. 2003;12(4):536-43. https://doi.org/10.1046/j.1365-2702.2003.00746.x.

6. Contro NA, Larson J, Scofield S, Sourkes B, Cohen HJ. Hospital staff and family perspectives regarding quality of pediatric palliative care. Pediatrics. 2004;114(5):1248-52. https://doi.org/10.1542/peds.2003-0857-L.

7. Engler AJ, Cusson RM, Brockett RT, et al. Neonatal staff and advanced practice nurses' perceptions of bereavement/end-of-life care of families of critically ill and/or dying infants. Am J Crit Care. 2004;13(6):489-98. https://doi.org/10.4037/ajcc2004.13.6.489.

8. Wool C, Catlin A. Perinatal bereavement and palliative care offered throughout the healthcare system. Ann Palliat Med. 2019;8(Suppl 1):22-9. https://doi.org/10.21037/apm.2018.11.03.

9. Almeida FD, Moraes MS, Cunha ML. Taking care of the newborn dying and their families: Nurses' experiences of neonatal intensive care. Rev Esc Enferm USP. 2016;50(n.esp):118-24. https://doi.org/10.1590/S0080-623420160000300018.

10. Ben-Ezra M, Palgi Y, Walker R, Many A, Hamam-Raz Y. The impact of perinatal death on obstetrics nurses: a longitudinal and crosssectional examination. J Perinat Med. 2014;42(1):75-81. https://doi.org/10.1515/jpm-2013-0071.

11. Mitchell M. Preparing student midwives to care for bereaved parents. Nurse Educ Pract. 2005;5(2):78-83. https://doi.org/10.1016/j.nepr.2004.03.005.

12. Wallbank S, Robertson N. Midwife and nurse responses to miscarriage, stillbirth and neonatal death: A critical review of qualitative research. Evidence-based Midwifery. 2008;6(3):100-7.

13. Wallbank S, Robertson N. Predictors of staff distress in response to professionally experienced miscarriage, stillbirth and neonatal loss: a questionnaire survey. Int J Nurs Stud. 2013;50(8):1090-7. https://doi.org/10.1016/j.ijnurstu.2012.11.022.

14. Chan MF, Chan SH, Day MC. A pilot study on nurses' attitudes toward perinatal bereavement support: a cluster analysis. Nurse Educ Today. 2004;24(3):202-10. https://doi.org/10.1016/j.nedt.2003.11.009.

15. Hamama-Raz Y, Walker RMA, Palgi Y, et al. Comorbidity of Post-traumatic Stress Symptoms and Depressive Symptoms among Obstetric Nurses with Perinatal Death Exposure. Isr J Psychiatry Relat Sci. 2016;53(2):58-63.

16. McCool W, Guidera M, Stenson M, Dauphinee L. The pain that binds us: midwives' experiences of loss and adverse outcomes around the world. Health Care Women Int. 2009;30(11):1003-13. https://doi.org/10.1080/07399330903134455.

17. DiMarco M, Renker P, Medas J, Bertosa H, Goranitis JL. Effects of an educational bereavement program on health care professionals' perceptions of perinatal loss. J Contin Educ Nurs. 2002;33(4):180-6. https://doi.org/10.3928/0022-0124-20020701-10.

18. Robinson M, Baker L, Nackerud L. The relationship of attachment theory and perinatal loss. Death Stud. 1999;23(3):257-70. https://doi.org/10.1080/074811899201073.

19. Jang SH. Neonatal Intensive Care Unit Nurses' Stress of End-of-Life Care for High-Risk Newborn. Seoul: Ewha Womans University; 2013.

20. World Health Organization. Process of translation and adaptation of instruments. http://www. who. int/substance_abuse/research_tools/translation/en/. Accessed July 16, 2020.

21. Jang HY, Jung JW. Delivery Room Nurses' Experiences of Caring of Stilbirth Babies and Their Parents. Korean J Women Health Nurs. 2017;23(3):166-80. https://doi.org/10.4069/kjwhn.2017.23.3.166.

22. Kingdon C, Givens JL, O'Donnell E, Turner M. Seeing and Holding Baby: Systematic Review of Clinical Management and Parental Outcomes After Stillbirth. Birth. 2015;42(3):206-18. https://doi.org/10.1111/birt.12176.

23. Chan MF, Lou FL, Arthur DG. A survey comparing the attitudes toward perinatal bereavement care of nurses from three Asian cities. Eval Health Prof. 2010;33(4):514-33. https://doi.org/10.1177/0163278710381092.

24. Kwon EH, Ju HO, Jeung EO, et al. Stress due to end-of-life care, coping strategies, and psychological well-being among nurses in neonatal intensive care units. Child Health Nurs Res. 2018;24(4):475-83.

25. Kim JH. The Job Stress, Perception, and Training Needs on the End-of-Life Care among Cancer Unit Nurses. Seoul: Yonsei University; 2009.

26. Kim KS. The Relationship among Perception of Death, End-of-life Care Stress and End-of-life Care Attitude of Nurses. Busan: Kosin University; 2016. 
27. Kim GH. The Perceptions of Hospice Palliative Care and Terminal Care Attitude of Nurses at Long-Term Care Hospitals. Busan: Catholic University of Pusan; 2016.

28. Kim S, No MJ, Moon KE, et al. Intensive care unit nurses' Death perception, End of life stress and end of life nursing attitudes. Journal of Korean Clinical Nursing Research. 2018;24(2):255-62. https://doi.org/10.22650/JKCNR.2018.24.2.255.

29. Wi DH, Kang SJ. Relationship Among Nurses' Knowledge, Attitude Towards Palliative Care and Perception of Death in Neonatal Intensive Care Units. Child Health Nurs Res. 2016;22(4):257-64. https://doi.org/10.4094/chnr.2016.22.4.257.

\section{Supplementary Files}

This is a list of supplementary files associated with this preprint. Click to download.

- supplementaryfile3.docx

- Supplementaryfile2.docx

- supplemenataryfile1.docx 